\title{
Thoracoplasty: A 15 Year Single Centre Experience
}

\author{
Dr.Dheeraj Sharma ${ }^{1}$,Dr. Anula Sisodia ${ }^{2}$,Dr. Sanjeev Devgarha ${ }^{3}$, \\ Dr. Rajendra Mohan Mathur ${ }^{4}$ \\ ${ }^{1}$ Assistant Professor, Department Of Paediatric Cardiac Surgery,Super Speciality Paediatric Hospital \&Post \\ Graduate Teaching Institute, Noida,Uttar Pradesh, India, \\ ${ }^{2}$ Assistant Professor, Department Of Cardiothoracic And Vascular Surgery, S.M.S Medical College, Jaipur, \\ Rajasthan, India \\ ${ }^{3}$ Professor, Department Of Cardiothoracic And Vascular Surgery, S.M.S Medical College, Jaipur, Rajasthan, \\ India \\ ${ }^{4}$ SeniorProfessor And Head, Department Of Cardiothoracic And Vascular Surgery, S.M.S Medical College, \\ Jaipur, Rajasthan, India
}

\begin{abstract}
:
Background:Thoracoplasty is removal of the skeletal support of the portion of chest allowing the chest wall to fall and fill the residual space. Thoracoplasty was originally described for the treatment to the sequel of pulmonary tuberculosis but later it was also considered for patients of chronic non bronchopleural fistulas secondary to many primary etiology. As far as tuberculosis is concerned after the advent of chemotherapy, the operation of thoracoplasty became nearly obsolete but its significance for the treatment of chronic non healing bronchopleural fistula has not reduced. Thus the significance of this surgery is quite high especially in developing countries where the burden of disease is still high despite the development of chemotherapy and where the incidence of post surgical bronchopleural fistula are high.

Methods: Between January 2000 and December 2014, we performed thoracoplasty in 569 patients at department of cardiothoracic surgery, S.M.S Medical College, Jaipur, Rajasthan, India. Regular follow up of patients was done and data analysed.

Results: Successful outcome in the form of control of sepsis, closure of bronchopleural fistula, sputum conversion and control of haemoptysis was achieved in most cases. There were 16 deaths in the entire series.

Conclusion:We conclude that thoracoplasty is still an operation of continued relevance in developing countries where the pulmonary tuberculosis is still prevalent despite the chemotherapeutic treatment and development of drug resistance, also the fact that these patients are severely debiliated and malnourished the incidence of development of bronchopulmonary fistula are much more. Thus thoracoplasty is not only related to treatment of sequele of pulmonary tuberculosis but also a vital tool in management of bronchoplieural fistula.
\end{abstract}

Keywords:Thoracoplasty, Tuberculosis, bronchopleural fistula.

\section{Introduction}

Today is the era of minimally invasive surgery and the advent of new technology has made it simpler for us. But with the development of minimally invasive surgery we are losing the art of conventional surgery, Thoracoplasty is one such art which has been catagorised as obsolete especially in western countries but developing countries like India, the original indication of this operation like pulmonary tuberculosis remains fairly prevalent and the condition is further complicated by rapid rise in drug resistant strains. Also the fact that most of these patients are severely debiliated and malnourished the incidence of development of bronchopulmonary fistula is much more. Thus thoracoplasty is not only related to treatment of sequele of pulmonary tuberculosis but also a vital tool in management of bronchopleural fistula. Hence, thoracoplasty is still a viable treatment option in this region of world.

\section{Patients and Methods .}

We present here our fifteen years experience of five hundred sixty-nine patients who underwent thoracoplasty. Between January 2000 and December 2014, 569 patients underwent thoracoplasty at department of Cardiothoracic surgery, S.M.S Medical College, Jaipur, Rajasthan, India . There were 404 men and 165 women whose age ranged from 15 years to 55 years (Mean age 33 years).

The indications of thoracoplasty in our series were same as with many other previously published studies and they were :

a. tubercular empyema (353 patients)

b. pyogenic empyema (91 patients) 
c. post-operative empyema with bronchopleural fistula (74 patients)

d. drug resistant pulmonary tuberculosis (40 patients)

e. post-treatment recurrent haemoptysis (11 patients).

Detailed informed consent was taken from the patient and relatives after properly explaining the procedure and its complications. All the patients were followed up during the stay in hospital and during outpatient department visits on regular interval through mails and telephonic conversations for upto 6 months, the follow up is nearly $95 \%$.

\section{Evaluation and surgical intervention:}

Patients who are taken for thoracoplasty were thoroughly and properly investigated in preoperative period. Preoperative workup included the routine blood investigations, bronchoscopic evaluation, chest Xray both PA and lateral views, computed tomography scan of thorax to look for condition of the diseased and contralateral lung, pulmonary function tests, assessment and improvement of the nutritional status, antibiotic treatment according to blood and pus culture report and in cases of pulmonart tuberculosis ATT was advised for atleast 3 months before taking these patients for surgery. Adequate drainage of empyema space was done in all cases by intercoastal chest tube drainage or by creating a pleurocutaneous window till the discharge becomes minimal.

As a departmental protocol we use double lumen endotracheal tube for endotracheal intubation with general anaesthesia in all cases as it prevents the spillage to other lung and allows selective deflation and inflation of the involved lung. Patient is placed in full lateral decubitus and thorax is opened on involved side by standard posterolateral thoracotomy. After thoracotomy the empyema cavity was entered by cutting the thickened pleura with electrocautry and only then the number and extent of rib excision was decided. The required ribs were excised from costochondral junction to the neck of the rib and the intercoastal muscles were allowed to fall back to fill the space. Back ends of the ribs were always resected completely. Occasionally, transverse processes were resected if deemed necessary. In patients with bronchopleural fistula a myoplastic flap created from intercostal muscles and adherent thickened pleura was sutured all around the fistula.In patients requiring surgery for control of upper zone cavity, the apex of the lung was freed and soft tissues were retracted downwards so as to obliterate the space (apicolysis) ,though an attempt was always made to preserve the first rib, it had to be removed in 132 cases to completely obliterate the apical space.

Two chest drains were placed one anteriorly and other posteriorly and the chest wall was closed in two layers with vicryl no 1 suture and skin approximated by interrupted mattress sutures by nylon 2-0. Compression dressing using rolls of cotton and bandage was done in all cases. The aim of compression was not only to minimize accumulation of blood but also to prevent any paradoxical movement of the chest wall in the postoperative period. Most of the patients were extubated in operating room but mechanical ventilation was necessary in 109 patients.

\section{Post-operative Management:}

Since the thoracotomy is the most painful incision it is our institutional policy to administer proper analgesia in form of narcotics at regular intervals. Physiotherapy in the form of active breathing, incentive spirometry and active shoulder movement was encouraged. Antibiotic regimen routinely consisted of cefoperazone with sulbactum, amikacin and meterogyl for atleast 7 days postoperatively. Daily cleaning and dressing were done and chest drains were kept for at least 10 days postoperatively even if the discharge and air leaks are minimal as a institutional policy.

\section{Results}

Out of 569 patients who underwent thoracoplasty, 16 patients died after surgery and 553 patients survived and out of these 553 patients 428 had an uneventful post-operative course. Fifty five patients had postoperative wound infection which increased morbidity (Table). In nine patients, there was a complete breakdown of the wound and recovery took about two months. In forty four patients empyema space persisted and a re-do thoracoplasty had to be resorted to. Sinuses continued to persist in seventeen patients, despite treatment. Seventy four patients who had post-operative bronchoplerual fistula (thirty four after lobectomy and fifteen after pneumonectomy and twenty five after decortication) had an uneventful post-operative course. As detailed earlier, intercostal muscle bundles were used to support the repair of the fistula. Air leak persisted for some time. However, in all the cases, fistulae closed completely and removal of the drainage tubes could be achieved in a period extending upto four weeks.

Two patients had bilateral pulmonary tuberculosis. Thoracoplasty involving resection of first to fifth ribs was carried out.Because of the bilateral nature of fibrotic nodulesthoracoplasty failed to control haemoptysis in these two cases.There were 16 deaths in the entire series (Table). There were 3 early deaths due respiratory failure and 
rest 13 deaths are late mortalities (after 30 days of surgery). All the late deaths were caused by persistent sepsis, septicemia and cachexia.

Table:Resultsofsurgeryin569patientswhounderwentthoracoplasty.

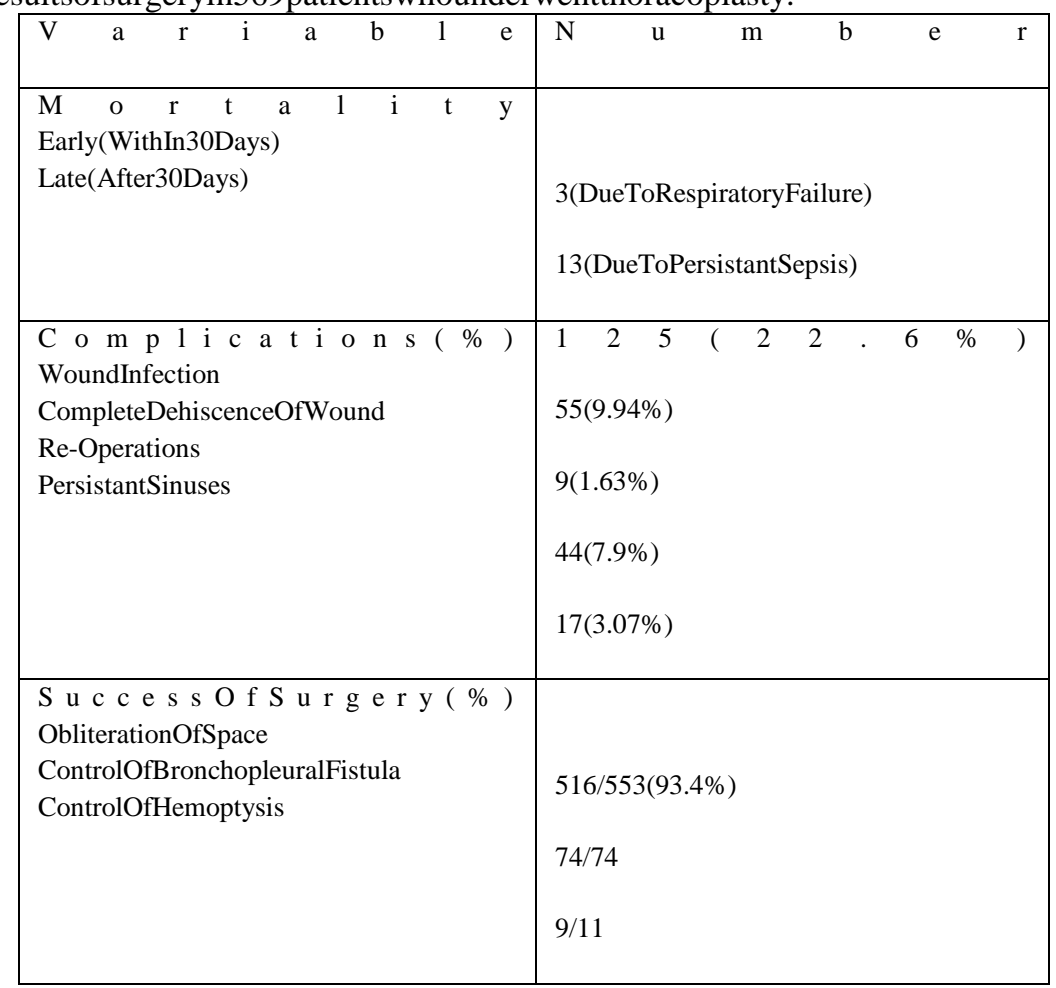

\section{Discussion}

The operative removal of the skeletal support of a portion of the chest is called thoracoplasty. The main aim of the surgery is to obliterate the persisting pleural space due to any etiology and it is usually accomplished by subperiosteal removal of varying number of rib segments to approximate the chest wall to the underlying lung or mediastinum ${ }^{2}$.

Thoracoplasty has evolved from many stages, starting from staged thoracoplasty procedure ( the Alexander procedure) which successfully collapsed the cavities of pulmonary tuberculosis and then came the Schede procedure which aims at reducing intrathoracic spaces or filling them with living tissue implants followed by Bjork osteoplastic thoracoplasty, tailoring thoracoplasty and Andrews thoracoplsty for chronic empyema with bronchopleural fistula. The indications for the operation of thoracoplasty are continuously decreasing as is evident from many published reports ${ }^{3,7}$ mainly due to development of effective chemotherapy for pulmonary tuberculosis and development of the various lung resection procedures, also the high morbidity and disfigurement are equally responsible for the reduced interest in this surgeryin developed countries. But the situation is not same in all parts of globe, there are regions like third world countries where the living conditions are not same as developed countries, where pulmonary tuberculosis and its complications are quite rampant, patient compliance is poor, drug resistance, malnutrition, poor health care delivery system and a certain amount of mismanagement at primary and secondary levels of health care. In these countries the operation of thoracoplasty is still required fairly often. As thoracoplasty is somewhat mutilating, it is always carried out as a last resort. In our institution, thoracoplasty is carried out for a few specific indications.

The operation is used for the treatment of chronic thoracic empyema when there is either insufficient or no remaining pulmonary tissue to obliterate the pleural space(destroyed underlying lung). If the lung cannot be brought to the chest wall [which is the objective of tube thoracostomy, rib resection and decortication), the chest wall must be brought to the lung (or the mediastinum if no lung exists) ${ }^{1,7}$. In 1985, Hopkins and Co-workers ${ }^{6}$ reviewed their experience with thoracoplasty and included in their indications for the procedure attempts to close persistent pleural spaces with and without infection and bronchial fistulae, both post-resection and in association with post pneumonic pleural suppuration. These indications are seen in limited numbers by western thoracic surgeons. Our experience suggests that a fairly large number of such patients exist in our community who are not suitable for decortication and could benefit from thoracoplasty.

As far as our study is concerned, our study is the largest case series presented till date and only other largest series available of recent time is from dewan and collegues ${ }^{8}$ who presented series of 139 cases in 1999. 
As far as results of our series are concerned they are well supported by facts and are in correlation with the results of various other studies till date.Today, thoracoplasty as an operation for management of chronic pleural empyema is accomplished in one stage ${ }^{2}$, because paradoxical chest wall motion is not a problem and chest wall are fixed and hence the problem of frail chest does not arise in the post-operative period. We have resected even upto 10 ribs in a single setting without any untoward effect. Although staging the procedure has been employed by other authors with equally good results, we do not consider it essential in all cases.

There is element of controversy regarding the removal of first rib during thoracoplasty. We always try to preserve the first rib for the fact that that it provides the structural support to the neck but in cases where there is upper lopbe cavity or space as seen mostly in post pneumonectomy cases first rib has to be removed as a part of apicolysis.. We have carried out removal of the first rib in seventeen cases as it was considered essential for complete space obliteration.

As far as drug resistant pulmonary tuberculosis are concerned they are primarily treated by chemotherapy and various forms of lung resections are adviced in cases of localized disease but thoracoplasty has a role in cases where the disease is generalized and associated with destroyed underlying lung.Cases of post treatment haemoptysis are best treated with lung resection if the disease is localized and the patient can tolerate lung resection. However, those patients who were judged to be unfit for resectional surgery were offered thoracoplasty with only partial success. In summary, we conclude that thoracoplasty is still an operation of continued relevance in developing countries where the pulmonary tuberculosis is still prevalent despite the chemotherapeutic treatment and development of drug resistance, also the fact that these patients are severely debiliated and malnourished the incidence of development of bronchopulmonary fistula are much more. Thus thoracoplasty is not only related to treatment of sequele of pulmonary tuberculosis but also a vital tool in management of bronchoplieural fistula.

We believe that this procedure will continue to be required at least in the foreseeable future. Conflict of interest: no conflict of interest of any author, non of the author received any grants Ethical approval: study does not involve use of any animal. And in case of patients all procedures performed in studies involving human participants were in accordance with the ethical standards of the institutional and/or national research committee and with the 1964 Helsinki declaration and its later amendments or comparable ethical standards. Written informed consent has been taken from patients and non has been forced to be a part of study.

\section{References}

[1]. Alexander J. The collapse therapy of pulmonary tuberculosis. 1937.

[2]. Barker WL. Thoracoplasty. Chest Surg Clin North Am 1994; 4: 593-615.

[3]. Peppas G, Molnar TF, Jeyasingham K, Kirk AB. Thoracoplasty in the context of current surgical practice. Ann Thorac Surg 1993; $56: 903-909$.

[4]. Pairolero PC, Trastek VF. Surgical management of chronic empyema : The role of thoracoplasty (Editorial). Ann Thorac Surg 1990; $50: 689-690$.

[5]. Jaretzki A (III). Role of thoracoplasty in the treatment of chronic empyema (Letter). Ann Thorac Surg 1991; 52: 584-585.

[6]. Horrigen TP, Snow NJ. Thoracoplasty : Current application to the infected pleural space. Ann Thorac Surg 1990; 50: 695-699.

[7]. Hopkins RA, Ungerleider RM, Status EW, et al. The modern use of thoracoplasty. Ann Thorac Surg 1985; 40: 181.

[8]. Dewan RK, Singh S, Kumar A, Meena BK. Thoracoplasty : An Obsolete Procedure ? .Indian J Chest Dis Allied Set 1999; 41 : 8388 